\title{
Treatment of Gastric Cancer
}

\author{
Stefano Rausei ${ }^{1, *(1) \text { and Georgios D. Lianos }}{ }^{2}$ \\ 1 Department of Surgery, ASST Valle Olona, Viale Eusebio Pastori 4, 21013 Gallarate, Italy \\ 2 Department of Surgery, University Hospital of Ioannina \& University of Ioannina, 45500 Ioannina, Greece; \\ georgiolianos@yahoo.gr \\ * Correspondence: stefano.rausei@gmail.com; Tel.: +39-349-853-5931
}

Received: 9 September 2020; Accepted: 14 September 2020; Published: 15 September 2020

Surgery represents the only method for potentially curative intent for gastric cancer (GC). However, even after surgery, the prognosis of locally advanced GC remains dramatically poor. Five-year survival rates with resection alone range from $23 \%$ to $49 \%$ among Western and approximately to $70 \%$ in the Eastern countries [1,2]. These variations have been attributed to several differences between East and West regarding cancer biology, earlier diagnosis through screening programs, and surgeons' compliance for a more extended surgery [3].

During the last two decades, it has been gradually accepted by the scientific community that GC patients should be treated by a multimodal and individualized approach [4]. However, the definition of the 'optimal' approach for GC patients is still debated and under extensive discussion.

Firstly, the treatment of GC has undoubtedly evolved with the recognition of the prognostic value of extended surgery, in terms of lymphadenectomy and multivisceral resection as well. On the other side, patient-friendly minimally invasive approaches along with enhanced recovery postoperative protocols have attracted scientific attention in GC management.

What is more, in the current century, randomized clinical trials demonstrated that chemotherapy or chemoradiation administered before, after, or both before and after surgery improve prognosis of GC patients [4].

After the long-debated discussion in the last century about the efficacy of extended lymphadenectomy, the results of a Taiwanese trial, published in 2006, have seemed rigorous enough to be considered definitive [5], along with the amount of consistent (though less strong) previous studies.

Now, the latest improvements in surgical techniques and increased surgeon experience make a patient-friendly minimally invasive surgical approach an achievable target also in GC treatment [6,7] and the surgical feasibility and safety of laparoscopic D2 distal gastrectomy have been demonstrated $[8,9]$. However, surgical outcomes (and technical perplexities) of laparoscopic total gastrectomy remain highly controversial $[7,10]$. Therefore, waiting for definite evidence of minimally invasive surgery for the cure of GC, its advent, along with its postoperative benefits-further enhanced by fast-track protocols-stressed a shift towards more personalized treatment of GC, with the intention of achieving better treatment efficacy for each patient, for each tumor [7].

Actually, "personalized treatment" and "patients' selection" are the key words for multimodal strategy to GC. In the 2000s, upfront surgery is no longer the gold standard for non-early cases. In the West, every locally advanced tumor (often assessed by staging laparoscopy) is an indication of neoadjuvant chemotherapy [4,11]; recently, even the far East introduced the neoadjuvant approach for every tumor with bulky nodes [12].

Two European prospective randomized trials (MAGIC, French FNCLCC/FFCD) comparing perioperative chemotherapy versus surgery alone have definitively introduced in the West this new important perspective of treatment for GC $[13,14]$. Additionally, the recent results from the FLOT4 trial [15] report that a FLOT regimen can be used as a safe and efficient new standard regimen in 
the perioperative treatment of resectable GC. Consistently with these data and with the efficacy demonstrated for S-1-based schedules in the adjuvant setting, almost surprisingly, in the East, the SPIRITS study showed the possibility to candidate to curative surgery patients affected by GC with extensive nodal involvement after preoperative chemotherapy [16].

It is out of doubt that there is a lot of experience nowadays with neoadjuvant therapy and the neoadjuvant concept has become widely accepted; most research is now focused on the identification of the best regimen. However, we feel cautious acceptance might be more prudent [17].

On other hand, it is not to be excluded that even some metastatic GCs could sensationally respond to a first-line chemotherapy up to a successful, curative conversion surgery [18].

Actually, the future success of any therapeutic strategy resides in anticipating the response to treatment and more generally, prognosis of GC patients. Molecular classification of GC could provide potential novel pathways for patient stratification and trials of targeted therapies aiming to improve survival from this deadly disease [19].

While possibly, the 'match' against GC is definitely playing in molecular laboratories, all physicians are continuously investing time and resources to add quantity and quality to the life of every patient. This Special Issue aims to explore the areas of GC treatment remodeled or reconsidered with this perspective in the last two decades. Intriguing molecular insights, innovative drugs, new surgical conquests (and reconquests), technological advances, and modern clinical approaches are some of the topics we aim to focus on.

Funding: This research received no external funding.

Conflicts of Interest: The authors declare no conflict of interest.

\section{References}

1. Bray, F.; Ferlay, J.; Soerjomataram, I.; Siegel, R.L.; Torre, L.A.; Jemal, A. Global cancer statistics 2018: GLOBOCAN estimates of incidence and mortality worldwide for 36 cancers in 185 countries. CA Cancer J. Clin. 2018, 68, 394-424. [CrossRef] [PubMed]

2. Fornaro, L.; Vasile, E.; Aprile, G.; Oliver Goetze, T.; Vivaldi, C.; Falcone, A.; Al-Batran, S.-E. Locally advanced gastro-oesophageal cancer: Recent therapeutic advances and research directions. Cancer Treat. Rev. 2018, 69, 90-100. [CrossRef] [PubMed]

3. Russo, A.; Li, P.; Strong, V.E. Differences in the multimodal treatment of gastric cancer: East versus west. J. Surg. Oncol. 2017, 9999, 1-12. [CrossRef] [PubMed]

4. Smyth, E.C.; Verheij, M.; Allum, W.; Cunningham, D.; Cervantes, A.; Arnold, D. Gastric cancer: ESMO Clinical Practice Guidelines for diagnosis, treatment and follow-up. Ann. Oncol. 2016, 27, v38-v49. [CrossRef] [PubMed]

5. Wu, C.-W.; Hsiung, C.A.; Lo, S.-S.; Hsieh, M.-C.; Chen, J.-H.; Li, A.F.-Y.; Lui, W.-Y.; Whang-Peng, J. Nodal dissection for patients with gastric cancer: A randomised controlled trial. Lancet Oncol. 2006, 7, 309-315. [CrossRef]

6. Lianos, G.D.; Rausei, S.; Ruspi, L.; Galli, F.; Mangano, A.; Roukos, D.H.; Dionigi, G.; Boni, L. Laparoscopic gastrectomy for gastric cancer: Current evidences. Int. J. Surg. 2014, 12, 1369-1373. [CrossRef] [PubMed]

7. Lianos, G.D.; Hasemaki, N.; Glantzounis, G.K.; Mitsis, M.; Rausei, S. Assessing safety and feasibility of 'pure' laparoscopic total gastrectomy for advanced gastric cancer in the West. Review article. Int. J. Surg. 2018, 53, 275-278. [CrossRef] [PubMed]

8. Kim, H.-H.; Han, S.-U.; Kim, M.-C.; Kim, W.; Lee, H.-J.; Ryu, S.W.; Cho, G.S.; Kim, C.Y.; Yang, D.J.; Song, K.Y.; et al. Effect of Laparoscopic Distal Gastrectomy vs. Open Distal Gastrectomy on Long-term Survival Among Patients with Stage I Gastric Cancer: The KLASS-01 Randomized Clinical Trial; Korean Laparoendoscopic Gastrointestinal Surgery Study (KLASS) Group. JAMA Oncol. 2019, 5, 506-513. [CrossRef] [PubMed]

9. Hyung, W.J.; Yang, H.-K.; Park, Y.-K.; Lee, H.-J.; An, J.Y.; Kim, W.; Kim, H.-I.; Kim, H.H.; Ryu, S.W.; Hur, H.; et al. Long-Term Outcomes of Laparoscopic Distal Gastrectomy for Locally Advanced Gastric Cancer: The KLASS-02-RCT Randomized Clinical Trial. J. Clin. Oncol. 2020, 20, JCO2001210. [CrossRef] [PubMed] 
10. Trapani, R.; Rausei, S.; Reddavid, R.; Degiuli, M.; ITALIAN RESEARCH GROUP FOR GASTRIC CANCER (GIRCG) Clinical Investigators. Risk factors for esophago-jejunal anastomosis leakage after total gastrectomy for cancer. A multicenter retrospective study of the Italian research group for gastric cancer. Eur. J. Surg. Oncol. 2020. [CrossRef] [PubMed]

11. National Comprehensive Cancer Network. Clinical Practice Guidelines in Oncology (NCCN Guidelines ${ }^{\circledR}$ ). Gastric Cancer. Version 3.2020. Available online: https://www.nccn.org/store/login/login.aspx?ReturnURL= https://www.nccn.org/professionals/physician_gls/pdf/gastric.pdf (accessed on 8 September 2020).

12. Japanese Gastric Cancer Association. Japanese gastric cancer treatment guidelines 2018 (5th edition). Gastric Cancer 2020, 1-21. [CrossRef]

13. Cunningham, D.; Allum, W.H.; Stenning, S.P.; Thompson, J.N.; Van de Velde, C.J.; Nicolson, M.; Scarffe, J.H.; Lofts, F.J.; Falk, S.J.; Iveson, T.J.; et al. Perioperative chemotherapy versus surgery alone for resectable gastroesophageal cancer. N. Engl. J. Med. 2006, 355, 11-20. [CrossRef] [PubMed]

14. Ychou, M.; Boige, V.; Pignon, J.P.; Conroy, T.; Bouché, O.; Lebreton, G.; Ducourtieux, M.; Bedenne, L.; Fabre, J.M.; Saint-Aubert, B.; et al. Perioperative chemotherapy compared with surgery alone for resectable gastroesophageal adenocarcinoma: An FNCLCC and FFCD multicenter phase III trial. J. Clin. Oncol. 2011, 29, 1715-1721. [CrossRef] [PubMed]

15. Al-Batran, S.E.; Homann, N.; Pauligk, C.; Goetze, T.O.; Meiler, J.; Kasper, S.; Kopp, H.G.; Mayer, F.; Haag, G.M.; Luley, K.; et al. FLOT4-AIO Investigators. Perioperative chemotherapy with fluorouracil plus leucovorin, oxaliplatin, and docetaxel versus fluorouracil or capecitabine plus cisplatin and epirubicin for locally advanced, resectable gastric or gastro-oesophageal junction adenocarcinoma (FLOT4): A randomised, phase 2/3 trial. Lancet 2019, 393, 1948-1957. [PubMed]

16. Koizumi, W.; Narahara, H.; Hara, T.; Takagane, A.; Akiya, T.; Takagi, M.; Miyashita, K.; Nishizaki, T.; Kobayashi, O.; Takiyama, W.; et al. S-1 plus cisplatin versus S-1 alone for first-line treatment of advanced gastric cancer (SPIRITS trial): A phase III trial. Lancet Oncol. 2008, 9, 215-221. [CrossRef]

17. Rausei, S.; Bali, C.; Lianos, G.D. Neoadjuvant chemotherapy for gastric cancer. Has the time to decelerate the enthusiasm passed us by? Semin. Oncol. 2020. [CrossRef] [PubMed]

18. Yoshida, K.; Yamaguchi, K.; Okumura, N.; Tanahashi, T.; Kodera, Y. Is conversion therapy possible in stage IV gastric cancer: The proposal of new biological categories of classification. Gastric Cancer 2016, 19, 329-338. [CrossRef] [PubMed]

19. The Cancer Genome Atlas Research Network. Comprehensive molecular characterization of gastric adenocarcinoma. Nature 2014, 513, 202-209. [CrossRef] [PubMed] 